\title{
Maladies orphelines: des paroles et des actes
}

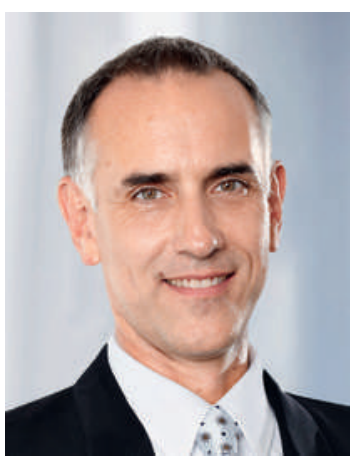

Probablement avant la pause estivale, l'Office fédéral de la santé publique (OFSP) devrait présenter son projet de «Concept pour les maladies rares», dont le but est de répondre au postulat de la conseillère nationale Ruth Humbel du 16 décembre 2010 pour «une stratégie nationale pour améliorer la situation médicale des personnes souffrant de maladies rares». Aux côtés d'acteurs de premier plan, la FMH s'engage activement depuis le début dans la communauté d'intérêts pour les maladies orphelines (CIMO) créée en août 2011 dans l'intention de conférer davantage de poids aux revendications en faveur d'un plan de mesures national. l'encouragement de la recherche et de la formation ainsi que le financement des mesures.

Dans le cadre de ces quatre ateliers, l'OFSP a également montré sa volonté de tenir les rênes du projet, mission qui relève bien évidemment de la souveraineté politique de la Confédération. Mais pour devenir un instrument efficace, le plan de mesures visé doit bénéficier d'un large soutien. C'est en effet le seul moyen d'agir positivement sur la situation des personnes atteintes de maladies orphelines et d'améliorer les conditions de prise en charge spécialisée, entre autres par les médecins. Il s'agit donc d'associer activement et directement au processus en cours toutes les organisations concernées et de prendre leur point de vue au sérieux.

La réussite d'un projet tel que le programme allemand $\mathrm{NAMSE}^{*}$ pose les premières pierres de ce que pourrait être la collaboration entre l'OFSP et les organisations de la CIMO. En Allemagne, le Ministère de la Santé, le Ministère de l'Educa-

\section{Associer activement et directement au processus en cours les personnes concernées et les experts chargés des soins et prendre leur point de vue au sérieux.}

Un chemin tortueux sépare le dépôt du postulat de la phase préconceptuelle d'aujourd'hui. Après une longue période de calme plat de la part des instances officielles, l'OFSP a convoqué d'autres organisations concernées et la CIMO à quatre ateliers entre l'automne 2013 et le printemps 2014. Dans les objectifs, les domaines de compétences et les priorités énoncés, l'OFSP reprend la plupart des points du plan de mesures que la CIMO lui a soumis en 2012. Plusieurs approches présentées par l'OFSP nous semblent cependant encourageantes.

\section{L'impatience que peut susciter l'attente du concept de I'OFSP ne suffira pas, à elle seule, à régler les problèmes des personnes concernées.}

Ainsi, nul ne conteste que les ressources et les compétences doivent être réunies au sein de centres de compétences dédiés aux différentes maladies orphelines, ni que l'accès aux diagnostics soit amélioré ou encore que l'échange d'informations et le transfert de connaissances ainsi que la collaboration internationale soient renforcés.

Cependant, les propositions de l'OFSP restent vagues sur un grand nombre de questions éminemment importantes pour les personnes concernées - en particulier l'égalité dans l'accès aux soins, le remboursement des traitements, la réglementation concernant les transferts de cas de l'AI à la LAMal, tion et de la Recherche, et les représentants des communautés d'intérêts collaborent en partenariat, et par conséquent sur le même pied d'égalité. NAMSE a permis de formuler un consensus de 52 propositions concrètes visant à améliorer la santé des personnes atteintes de maladies orphelines ont été reprises dans un plan d'action national. Si la Suisse poursuit son engagement, il serait souhaitable de miser également sur une coopération encore plus étroite que par le passé entre les organisations impliquées.

En effet, l'impatience que peut susciter l'attente du concept de l'OFSP ne suffira pas, à elle seule, à régler les problèmes les plus prioritaires des personnes concernées et de leurs proches. Il est à prévoir que la mise en œuvre concrète des différentes mesures sera elle aussi jalonnée de nombreux obstacles. Il est cependant possible de les surmonter si tous les acteurs manifestent leur volonté de collaborer en partenariat. C'est la condition sine qua non pour réaliser rapidement des améliorations substantielles et durables pour toutes les personnes concernées. Leur souffrance et la nécessité d'agir, n'ayant pas baissé en intensité, ne tolèrent plus de retards.

Gert Printzen, membre du Comité central de la FMH, responsable du département eHealth - informatique et documentation médicales / produits thérapeutiques

* NAMSE: Ligue d'action nationale pour les personnes atteintes de maladies orphelines (Nationales Aktionsbündnis für Menschen mit Seltenen Erkrankungen) 